\title{
Possible Detection of p-mode Oscillations on Procyon ${ }^{1}$
}

\author{
C. Barban, E. Michel
}

Observatoire de Paris, DASGAL, UMR 235, 92195 Meudon, France

M. Martic, J. Schmitt, F. Bouchy, J.C. Lebrun, P. Connes, J.L. Bertaux CNRS, Service d'Aéronomie, 91371 Verrières le Buisson, France

\author{
A. Baglin \\ Observatoire de Paris, DESPA, URA 264, 92195 Meudon, France
}

\begin{abstract}
We have observed Procyon with the fiber-fed échelle spectrograph ELODIE coupled with a Fabry-Perot etalon at the 193-cm of the Observatoire de Haute Provence (France) over 5 nights during 1997 December - 1998 January. Here, we present the results of a search for solar-like oscillations on this star and the performance of this instrumentation for asteroseismology. The power spectra show an excess of signal between $0.42 \mathrm{mHz}$ and $1.46 \mathrm{mHz}$, which could be due to stellar oscillations.
\end{abstract}

\section{Introduction}

Observations of solar p-mode oscillations have resulted in great progress in understanding the internal structure of the Sun. A detection of solar-like oscillations (p-modes) on other stars will provide new constraints for internal structure and stellar evolution models.

According to theoretical estimations (Houdek et al. 1995), solar-like oscillations should be present in $\mathrm{F}$ to $\mathrm{K}$ stars on the main sequence with a maximum amplitude of about $2 \mathrm{~m} / \mathrm{s}$ for early F stars (F0-F3). We choose to observe Procyon because of its proximity, its spectral type and its low rotational velocity (F5 IV-V, $m_{V}=0.34, v \sin i=3$ to $6 \mathrm{~km} / \mathrm{s}$ ). For this star, we expect oscillations with a maximum amplitude of around $1-1.5 \mathrm{~m} / \mathrm{s}$ around $1 \mathrm{mHz}$ (Houdek et al. 1995, Kjeldsen et al. 1995). Up to now, no unambiguous detection of solar-like oscillations on Procyon has been confirmed.

\footnotetext{
${ }^{1}$ Based on observations collected at the Observatoire de Haute-Provence (CNRS, France).
} 


\section{Observations}

We observed Procyon on 5 nights during 1997 December - 1998 January with the spectrograph ELODIE at the 193-cm telescope of the Observatoire de HauteProvence (OHP), France. Due to poor weather, we obtained only $17.9 \mathrm{~h}$ of useable data, over 3 nights. ELODIE is a fiber-fed échelle cross-dispersion spectrograph: $R=40000,3900-6800 \AA, 67$ orders, $1024 \times 1024$ CCD detector (Baranne et al. 1996). A double-scrambler has been installed since 1997. For the high precision required for these measurements, it is necessary to control the drifts of the instrument. For this, we used a Zerodur-spacer Fabry-Perot (FP) etalon, illuminated by a white light source and stabilized in temperature. The periodic channelled spectrum, over the whole wavelength range, given by the FP has been shown to be the best possible reference to detect the instrumental errors on short term periods (i.e on the order of several hours) (Connes 1985). The spectrum of the star and the spectrum of the FP are recorded simultaneously. Orders for both spectra alternate on the CCD.

\section{Data reduction}

We search for stellar oscillations from the radial velocity variations. The star and FP radial velocity shifts $\left(\Delta v_{i}(\operatorname{star}, t)\right.$ and $\left.\Delta v_{i}(\mathrm{FP}, t)\right)$, for each échelle order $i$ are given by the spectral shift of each spectrum (star and FP) relative to a reference spectrum (star and $\mathrm{FP}$ ). The reference is the best $\mathrm{S} / \mathrm{N}$ spectrum of the star and the one of the FP obtained during the night. We used all the spectral lines to determine the radial-velocity shifts. No calibration in wavelength is required since we do not calculate absolute radial velocities.

For each order $i$ of the échelle spectrograph, we correct the radial-velocity shifts observed for the star by the radial-velocity shifts of the FP, and by the computed radial velocity of the Earth:

$$
\Delta v_{i}(t)=\Delta v_{i}(\mathrm{star}, t)-\Delta v_{i}(\mathrm{FP}, t)-v(\text { Earth }, t) .
$$

For each night, after the corrections mentionned above, we calculated a weighted average of the radial velocity shifts for the orders not contaminated by telluric lines and from the 10th order (for Procyon, we used 47 orders):

$$
\Delta v(t)=\frac{\sum_{i} \frac{1}{\sigma_{i}} \Delta v_{i}(t)}{\sum_{i} \frac{1}{\sigma_{i}}}
$$

where $\sigma_{i}$ is the standard deviation of $\Delta v_{i}(t)$.

Figure 1 is an example of a time series obtained during this mission.

\section{Results}

\subsection{Performance of the instrumentation}

To estimate the performance of ELODIE+FP for asteroseismology, we analysed the 17.9-h data like a single time series with respect to time. We obtained: 


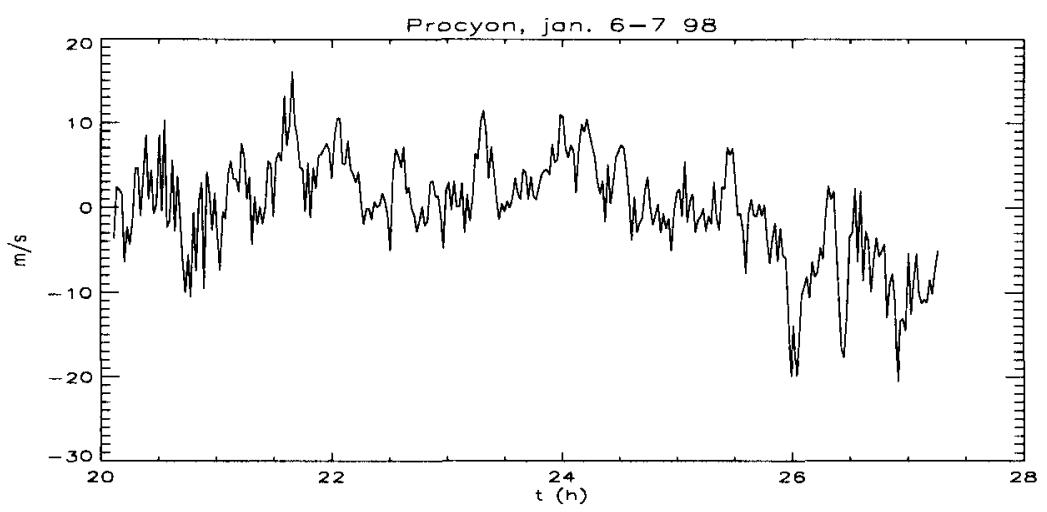

Figure 1. Weighted average of the radial velocity shifts over 47 orders as a function of time, obtained for Procyon, 1998 January 6-7 (the average has been removed to obtain a zero mean).

- a mean power noise level $\left\langle a^{2}\right\rangle$ of about $0.05(\mathrm{~m} / \mathrm{s})^{2}$ for $f \geq 1.5 \mathrm{mHz}$. The detection threshold $s$ for a single periodic oscillation corresponding to this mean power noise level is about $89 \mathrm{~cm} / \mathrm{s}$ with a confidence level of $99 \%$ $\left(\sqrt{\frac{s^{2}}{\left\langle a^{2}\right\rangle}} \geq 4\right)$.

- a standard deviation $\sigma \sim 3.16 \mathrm{~m} / \mathrm{s}$ for a 40 -s exposure time or $\sim 0.33 \mathrm{~m} / \mathrm{s}$ for a 3600 -s exposure time $\left(\sigma=\frac{\sqrt{\left(a^{2}\right\rangle} \sqrt{N}}{2}\right.$, where $N$ is the number of data points, $\sigma \propto \frac{1}{\sqrt{t}}$, and $t$ is the exposure time).

\subsection{Results obtained for Procyon}

Figure 2 represents the average of the 3 power spectra for the 3 useable nights on Procyon. The data are not filtered at low frequencies. No peaks nor excess power exceeding the noise level are observed for $f \geq 1.46 \mathrm{mHz}$. However, an excess power is clearly present between $0.42 \mathrm{mHz}$ and $1.46 \mathrm{mHz}$ with an amplitude of around $2(\mathrm{~m} / \mathrm{s})^{2}$; this will be presented in more details in Barban et al. (1998).

\section{Discussion}

\subsection{Comparison of noise levels for different observations}

A comparison of noise levels of Procyon observations obtained by ELODIE and by different instruments is shown in table 1 .

Brown and his coworkers used the Advanced Fiber Optic Echelle (AFOE) spectrograph (Brown et al. 1994), installed at the Tillinghast 1.5-m telescope at the F.L. Whipple Observatory, Arizona, USA. Radial velocity shifts are computed from all the spectrum lines accessible by AFOE (wavelength range: typically between 3920 and $6640 \AA$ over some 24 orders). 


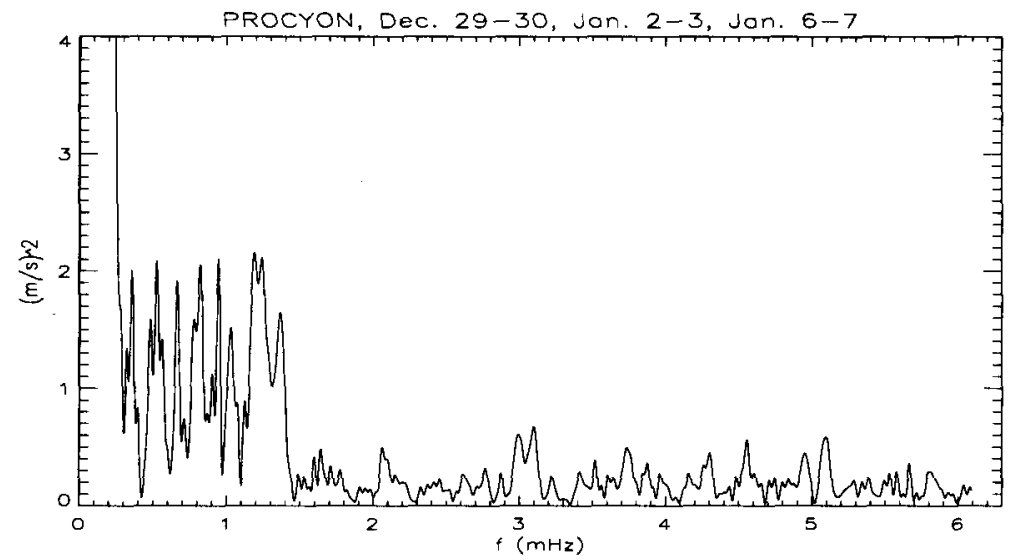

Figure 2. Average of the 3 power spectra of Procyon, corresponding to the 3 useable nights ( 47 orders used) (bin $\sim 4.3 \times 10^{-3} \mathrm{mHz}$, resolution $\sim 0.04 \mathrm{mHz}$ ).

Mosser and his coworkers observed Procyon in February 1998 with the Fourier Transform Spectrometer (FTS) based at the 3.6-m CFH telescope. They obtained $38 \mathrm{~h}$ of useable data. The FTS was used as an accelerometer in a new seismic mode. Radial velocities were calculated from only a dozen lines in the near infrared.

The $\sigma$ obtained with ELODIE+FP is comparable to that obtained by Brown et al. (1998), in spite of poor weather conditions during our observations.

Table 1. Procyon noise level obtained by different instruments ( $D$ : diameter of the telescope, $\sigma^{*}$ : standard deviation for a 1-h exposure).

\begin{tabular}{cccl} 
Instrument & $D(\mathrm{~m})$ & $\sigma^{*}(\mathrm{~m} / \mathrm{s})$ & Reference \\
\hline AFOE & 1.5 & $1.04^{\mathrm{a}}$ & Brown et al. (1994) \\
AFOE & 1.5 & 0.46 & Brown et al. (1998) \\
FTS & 3.6 & 1.66 & Mosser et al. (1998) \\
ELODIE+FP & 1.93 & 0.33 & this paper
\end{tabular}

${ }^{a}$ Procyon was observed on only one night.

\subsection{Search for solar-like oscillations on Procyon}

Mosser et al. (1998) did not detect any significant excess in the power spectrum near $1 \mathrm{mHz}$. Their limit on the amplitude of the modes does not exceed $1 \mathrm{~m} / \mathrm{s}$.

Brown et al. (1998) found an excess power similar to what we detected, in the same frequency domain and with a similar amplitude. 
Can we conclude that we have an actual detection of solar-like oscillations on Procyon? It is important to look for possible instrumental bias in our observations. We are scrutinizing the different sources of noise. In particular, periodic temperature variations of ELODIE's room gave components at the considered frequencies, although with a small amplitude. The study of this problem is in progress. The frequency domain and the amplitude of the excess power detected agree with the theoretical estimations which predict a signal around $1 \mathrm{mHz}$ with a maximum amplitude of around 1-1.5 m/s (Houdek et al. 1995 , Kjeldsen et al. 1995). The cut-off frequency observed around $1.46 \mathrm{mHz}$ is in agreement with these theoretical calculations. These single-site data are limited in frequency resolution; since the frequency separations are expected to be small, we do not expect to observe isolated peaks but rather an excess power similar to what is seen in our observations. And finally, the fact that two independent observations lead to a similar excess power in the power spectra leads us to think that it most likely has a stellar origin.

\section{Conclusion}

The noise level obtained from Procyon observations shows that ELODIE plus a FP etalon with the 193-cm telescope of the OHP is a well-suited instrument for asteroseismology. We obtained a detection threshold for a single periodic oscillation of about $89 \mathrm{~cm} / \mathrm{s}$ with a confidence level of $99 \%$ over $17.9 \mathrm{~h}$ of observations.

We detect an excess power between $0.42 \mathrm{mHz}$ and $1.46 \mathrm{mHz}$ with an amplitude of around $2(\mathrm{~m} / \mathrm{s})^{2}$ on Procyon. This excess power could be associated with stellar oscillations.

As mentionned above, these single-site data may not be sufficient for clear identification of individual modes. We will observe Procyon in 1998 November and probably in 1999 February; combined observations are welcomed! A two-site campaign is being prepared with Brown and his coworkers for 1999 February. Both instruments are capable of providing Doppler measurements with the required precision of a few $\mathrm{m} / \mathrm{s}$, and the $\sim 7$ hour longitude separation between them allows the acquisition of relatively long uninterrupted data strings.

Acknowledgments. We would like to thank the direction of OHP, and the technical staff of the 193-cm telescope, for their assistance and their efforts to adapt our instrumentation to the telescope which allowed us to obtain the data described here.

\section{Discussion}

Noyes: This is very beautiful work. It is likely that the AFOE can be scheduled in coordination with this instrument for two-thirds of a 3-station network to observe Procyon with nearly complete 24-hour coverage. It remains for us to find an interested group capable of making precise radial-velocity observations of Procyon with precision in the $\mathrm{m} / \mathrm{s}$ range, and located at a longitude complementary to both OHP and to Tucson, AZ. If successful observations can be 
carried out, this holds great promise of allowing, finally, definitive detection and identification of p-mode oscillations in another sun-like star.

\section{References}

Baranne A., Queloz D., Mayor M., Adrianzyk G., Knispel G., Kohler D., Lacroix D., Meunier J.P., Rimbaud G. \&Vin A., 1996, A\&AS, 119, 373

Barban C., Martic M., Schmitt J., Michel E., Bouchy F., Connes P., Lebrun J.C., Baglin A. \& Bertaux J.L., 1998, in preparation

Brown, T.M., Noyes R.W., Nisenson P. \& Korzennik S.G., 1994, PASP106, 1285

Brown, T.M., et al., 1999, in Proc. IAU colloquiun 170: Precise stellar radial velocities, ed. J.B. Hearnshaw and C.D. Scarfe, Conference series, ASP, to be published.

Connes P., 1985, Ap\&SS, 110, 211

Houdek, G., Rogl, J., Balmforth, N. \& Christensen-Dasgaard, J., 1995, in Proc. GONG '94: Helio- and astero- seismology from Earth and Space, Ulrich, R.K., Rhodes Jr, E.J., Däppen, W., PASC 76, San Francisco, 641

Kjeldsen, H., Bedding, T.R., 1995, A\&A, 293, 87

Mosser, B., Maillard, J.P., Mékarnia, D., Gay, J., 1998, submitted to A\&A 